\title{
Vereine als Schulen des Vertrauens? Eine empirische Analyse zur Zivilgesellschaft in der Schweiz
}

\author{
Markus Freitag, Nicolas Grießhaber und Richard Traunmüller \\ Universität Konstanz
}

\begin{abstract}
In der Tradition von Alexis de Tocqueville wird zivilgesellschaftlichen Vereinigungen eine essentielle Bedeutung in der zwischenmenschlichen Vertrauensbildung zugeschrieben. Ziel des vorliegenden Beitrages ist es, den Zusammenhang zwischen Vereinsengagement und generalisiertem Vertrauen differenzierter $z u$ betrachten und empirisch für die Schweiz zu untersuchen. Dabei werden sowohl das Engagement in verschiedenen Organisationstypen (brückenbildende vs. abgrenzende und isolierte vs. verbundene) als auch die vereinstypusunabhängige Integration miteinander verglichen. Empirische Analysen auf Basis aktueller Daten des Schweizer FreiwilligenMonitors 2007 kommen dabei zu dem Schluss, dass entgegen weitverbreiteter Meinung die Vertrauensentwicklung in der Schweiz weniger vom Engagement in spezifischen Vereinen abhängt, sondern vielmehr dem Ausmass der persönlichen Integrationsbereitschaft geschuldet ist. Von entscheidender Bedeutung für die Vertrauensentwicklung ist also nicht die Qualität der hier untersuchten einzelnen Vereinigungen, sondern die Quantität des persönlichen Engagements.
\end{abstract}

KEywords: Social Capital • Civil Society • Social Trust • Associational Life • Switzerland

\section{Einleitung}

Vertrauen steht gegenwärtig im Mittelpunkt vielfältiger Debatten. Während aus politikwissenschaftlicher Perspektive die Wirkung des Ver-

\footnotetext{
1 Dieser Beitrag ist im Rahmen eines von der Deutschen Forschungsgemeinschaft (DFG) durch das Exzellenzcluster 16 "Kulturelle Grundlagen von Integration" unterstützten Projektes zu den Sozialkapitalwelten im interkulturellen Vergleich und innerhalb der von der Schweizerischen Gemeinnützigen Gesellschaft (SGG) finanzierten Forschungsstelle "Bürgerschaftliches Engagement und Sozialkapital" entstanden. Wir danken den Herausgebern und den beiden anonymen Gutachtern für ihre kritischen und hilfreichen Anmerkungen sowie Birgit Jacob für die Hilfe bei der Endredaktion des Manuskriptes.
}

Konstanzer Online-Publikations-System (KOPS)

URN: http://nbn-resolving.de/urn:nbn:de:bsz:352-opus-109235

URL: http://kops.ub.uni-konstanz.de/volltexte/2010/10923 
trauens auf die Funktions- und Leistungsfähigkeit von Demokratien im Vordergrund steht, werden von soziologischer Seite vor allem die sozialintegrativen Leistungen des Vertrauens in zunehmend individualisierten Gesellschaften herausgestellt. Aus wirtschaftswissenschaftlicher Sicht wird der Stellenwert des Vertrauens in der Senkung marktwirtschaftlicher und unternehmerischer Transaktionskosten gesehen. In unserer alltäglichen Lebenswelt wiederum begegnet uns der Aspekt des Vertrauens immer dann, wenn die Risikogesellschaft ihren Tribut fordert. Eltern, Freunde oder Medien ermahnen uns, im täglichen Leben Vorsicht walten zu lassen und gegebenenfalls fremden Menschen, Politikern oder unseren Anlageberatern nicht blindlings zu vertrauen. Die global vernetzte Wirtschaft mit ihren Begleiterscheinungen einer wachsenden Mobilität, neuer Kommunikationstechnologien und einer Erhöhung der Arbeitsteilung akzentuiert diese Gefühle der Unsicherheit und Ungewissheit und fordert von uns die Kooperation mit persönlich unbekannten Menschen ein, die eines Vertrauensvorschusses bedürfen.

Angesichts der rundum diskussionslosen Relevanz zwischenmenschlichen Vertrauens stellt sich umso dringlicher die Frage, wie dieses kostbare Gut erzeugt werden kann. Auf der Suche nach den Ursprüngen generalisierten Vertrauens verorten viele dessen Wurzeln in gesellschaftlichen Entwicklungspfaden und fortdauernden, fest verankerten kulturellen $\mathrm{Mu}-$ stern sozialer Interaktion (Fukuyama 1995; Hooghe/Stolle 2003; Putnam 1993, 2000): Im Anschluss an diesen gesellschaftszentrierten Ansatz wird zwischenmenschliches Vertrauen auf eine als organisch charakterisierbare Art durch lange historische Prozesse geformt. Es sind insbesondere weit zurückreichende Traditionen zivilgesellschaftlichen Engagements, die generalisiertes Vertrauen nach sich ziehen (Putnam 2000). In der Tradition von Alexis de Tocqueville wird davon ausgegangen, dass Netzwerke der Zivilgesellschaft, will sagen: Vereine und Freiwilligenorganisationen als "Schulen des Vertrauens" fungieren und die Entstehung von gemeinschaftlichen Werten sowie zwischenmenschliches Vertrauen fördern. Als regelmässige und auf Dauer angelegte Beziehungen bilden Vereine ein Umfeld, in dem die Mitglieder eine gemeinschaftsbezogene Kommunikations-, Kooperations- und Hilfsbereitschaft erlernen und diese zur Lösung kollektiver oder individueller Probleme einsetzen können (Lippl 2007; Putnam 1993: 90). Zusammengefasst sind Vereine und Freiwilligenorganisationen diesem "Tocqueville-Modell" zufolge "part of the social infrastructure of society that makes the generation of trust possible, and that at least makes 
it easier for trust relations and trusting attitudes to develop and to re-enforce themselves within the population" (Anheier/Kendall 2002: 344).

Obschon in dieser Blickrichtung argumentiert wird, dass die persönliche Vertrauensbildung insbesondere dem aktiven Vereinsengagement geschuldet ist, weisen jüngere Forschungen darauf hin, dass nicht jedes Vereinsengagement gleichermassen zum Aufbau des zwischenmenschlichen Vertrauens beiträgt (Paxton 2007; Putnam 2000; Stolle/Rochon 1998; Zmerli 2003). Vielmehr sei die Qualität von Vereinigungen, also der Vereinstypus ausschlaggebend. Zwei Anschauungen dominieren hier die Diskussion (Coffé/Geys 2007): Einer ersten Konzeption folgend werden Vereine in brückenbildende und abgrenzende Formen der Soziabilität eingeteilt, wobei vor allem ersteren eine vertrauensförderliche Wirkung zugeschrieben wird (Putnam 2000: 22-24; Zmerli 2003, 2008). Eine zweite Auslegung unterscheidet dagegen zwischen verbundenen und isolierten Organisationen, welche sich ebenfalls hinsichtlich ihres Potentials zur Vertrauensentwicklung unterscheiden sollten (Paxton 2002, 2007).

Die vorliegende Arbeit schliesst an diese Diskussion an und erörtert den Zusammenhang zwischen aktivem Engagement in verschiedenen Vereinstypen und generalisiertem Vertrauen in der Schweiz. Dabei werden sowohl mögliche Unterschiede zwischen Organisationstypen hinsichtlich der Heterogenität ihrer Mitglieder und ihrer Orientierung über die eigenen Organisationsgrenzen hinweg als auch die Breite individueller Interaktionen innerhalb dieser Netzwerke berücksichtigt. Wir stellen dabei die Annahme vereinstypischer Vertrauensgeneratoren in Abrede und argumentieren, dass eher das persönliche Ausmass an vereinsmässiger Involvierung - unabhängig vom Vereinstypus - den Grad der zwischenmenschlichen Vertrauensbildung systematisch beeinflusst. Kurzum: Statt der Qualität bestimmter Vereine als Vertrauensgeneratoren ist vielmehr die Quantität der persönlichen assoziativen Einbindung für die Entwicklung des zwischenmenschlichen Vertrauens ausschlaggebend. Unsere diesbezüglichen Analysen und Ergebnisse basieren auf der Erhebung im Rahmen des Schweizer Freiwilligen-Monitors aus dem Jahre 2006 (Stadelmann-Steffen et al. 2007).

Der Beitrag gliedert sich wie folgt: Zunächst soll die vermutete Beziehung verschiedener Aspekte aktiver Tätigkeit in Freiwilligenorganisationen mit der Ausbildung von Vertrauen theoretisch fundiert und zu testende Hypothesen bezüglich dieses Zusammenhangs abgeleitet werden. Hierbei kommen die unterschiedlichen Sichtweisen zur Sprache. Im Anschluss an eine genaue Vorstellung des methodischen Vorgehens und der Operationalisierung der verwendeten Variablen stehen die empirischen Befunde der 
Analyse im Zentrum dieser Arbeit. Abschliessend soll eine kritische Bewertung dieser Ergebnisse und ein Ausblick auf mögliche Anknüpfungspunkte für die weitere Erforschung des untersuchten Zusammenhangs gegeben werden.

\section{Theorie und Hypothesen zum Zusammenhang zwischen Vereins- engagement und generalisiertem Vertrauen}

Vertrauen kann ganz allgemein gesprochen als positive Erwartung umschrieben werden, "dass andere durch ihr Handeln oder Unterlassen zum Wohlergehen eines einzelnen oder einer Gruppe beitragen, jedenfalls von schädigenden Handlungen absehen" (Offe 2001: 249). Dabei stellt gerade generalisiertes Vertrauen, welches sich auf die Mitmenschen im Allgemeinen bezieht und somit auch jenseits unmittelbarer Bekanntschaft auf andere Mitbürger und Fremde erstreckt, eine wichtige soziale Ressource dar (vgl. Freitag/Traunmüller 2009; Stolle 2002; Uslaner 2002; Yamagishi/Yamagishi 1994). ${ }^{2}$ Als Glaube an die prinzipielle Wohlgesinntheit und Verlässlichkeit der meisten Mitmenschen ermutigt und erleichtert es individuelle wie kollektive Anstrengungen, welche ansonsten aus Furcht vor dem opportunistischen Verhalten anderer entweder nur unter sehr hohen Kosten zu bewerkstelligen wären oder einfach ganz unterlassen würden. Gesellschaften, deren Mitglieder sich als vertrauenswürdig erweisen und welche sich gegenseitig in hohem Masse vertrauen, können folglich weit mehr erreichen als vergleichbare Gesellschaften, denen es an Vertrauen mangelt (Coleman 1990: 304). In diesem Sinne überwindet Ver-

\footnotetext{
2 Im Gegensatz zum generalisierten Vertrauen oder Fremdvertrauen basiert das sogenannte partikulare Vertrauen oder Nahbereichsvertrauen auf der persönlichen Kenntnis der zu vertrauenden Personen und erstreckt sich so auf eine zu überblickende soziale Einheit (Familie, Freunde, Nachbarn, Arbeitskollegen). Diese Vertrauensbeziehungen beruhen auf geteilten Lebens- und Erfahrungswelten und auf zukünftig zu erwartenden sozialen Austauschprozessen zwischen den Vertrauenden. Grundlage des Vertrauens ist all das, was wir über andere wahrnehmbar in Erfahrung bringen. Vertrauen wird damit zum eng gestreuten kognitiven Phänomen. "Fremde" werden - wenn uberhaupt - nur dann in diesen Vertrauenskreis aufgenommen, sofern sie bestimmten vorab definierten Filterkriterien wie etwa Alter, Geschlecht, Religions- und Vereinszugehörigkeit oder Ethnizität genügen. Es bleibt festzuhalten, dass sich die sozialwissenschaftliche Forschungspraxis wie auch die vorliegende Studie aus Gründen der Datenverfügbarkeit in erster Linie mit der generalisierten Vertrauensform auseinandersetzt. Ausnahmen sind die Arbeiten von Freitag/Traunmüller (2009); Gibson (2001); Herreros (2004); Uslaner (2002) und Welch et al. (2007).
} 
trauen in andere Menschen das für soziale Beziehungen charakteristische Problem der Unsicherheit über die sequentielle Abfolge vereinbarter Austauschhandlungen. Vertrauen ist somit ein Mittel, welches Austauschprozesse reibungsloser macht und durch das dauerhafte und wechselseitig ertragreiche Kooperationsbeziehungen in Gang gesetzt werden können (vgl. Preisendörfer 1995; Uslaner 2002; Whiteley 2000).

Vor allem im Rahmen der prominenten Sozialkapitaltheorie wird dem generalisierten Vertrauen daher eine besondere Bedeutung beigemessen (Coleman 1990; Fukuyama 1995; Putnam 1993, 2000). ${ }^{3}$ Jenseits der Beschäftigung mit den vielfältigen positiven Wirkungen von Vertrauen nimmt in dieser Denkschule jedoch vor allem die Frage nach den Ursprüngen verbreiteter Vertrauenseinstellungen eine zentrale Stellung ein. Den eigentlichen "Kern der Sozialkapitaltheorie" stellt dabei die von Tocqueville inspirierte These dar, dass generalisiertes Vertrauen vornehmlich in den vielfältigen Vereins- und Organisationsstrukturen einer vitalen Zivilgesellschaft wurzelt, dort regeneriert und aufrechterhalten wird (Freitag 2003: 219; Hooghe/Stolle 2003; Lippl 2007; Putnam 1993, 2000). Vereine und Freiwilligenorganisationen fungieren in diesem Sinne als bedeutende "Schulen des Vertrauens".

Als Vermittler von gemeinschaftlichen Werten und Normen sowie als Opportunitätsstruktur für positive Erfahrungen kommt zivilgesellschaftlichen Vereinigungen eine wichtige sozialisatorische Funktion zu (Brehm/ Rahn 1997; Claibourn/Martin 2000; Hooghe/Stolle 2003; Putnam 2000). So fördern Vereinigungen das Vertrauen unter ihren Mitgliedern einerseits über in der Organisation verankerte Werte und Normen. Mitglieder verinnerlichen durch aktives Engagement innerhalb der Organisation deren grundlegende Verhaltensregeln und Vorstellungen über den Umgang mit anderen. Mitglieder von Freiwilligenorganisationen werden somit in demokratischere und sozialere Wertstrukturen sozialisiert und übertragen diese positiven Einstellungen und Menschenbilder auf die Gesellschaft im Allgemeinen (Hooghe 2003: 89). Andererseits stellen Vereine eine Arena für regelmässige Treffen und auf Dauer gestellte Austauschbeziehungen dar. Dies und die Gewissheit, dass einheitliche gesellschaftliche Werte in der Gruppe bestehen und abweichende Handlungen negativ sanktioniert

3 In der Tat wird hohes Vertrauen mit zahlreichen positiven Wirkungen, wie etwa stabiler Demokratie und effektiven administrativen Institutionen, hohem Wirtschaftswachstum, technologischem Innovationspotential, öffentlicher Gesundheit sowie subjektivem Wohlbefinden in Verbindung gebracht (Putnam 2000). Umgekehrt führt ein Mangel an Vertrauen zu einer Vielfalt sozialer, ökonomischer und politischer Fehlentwicklungen. 
werden, schafft Sicherheit bezüglich der Vertrauenswürdigkeit anderer (Paxton 2007: 50). Mitglieder haben daher die Möglichkeit, positive Erfahrungen im Umgang mit anderen zu sammeln und zu lernen, dass Kooperation sich auszahlt, was sich wiederum auf zukünftige Kooperationsentscheidungen auswirkt (Levi 1998: 85). Mitglieder lernen dabei jedoch nicht nur, den Mitgliedern der eigenen Organisation zu vertrauen, sondern entwickeln gleichzeitig ein generalisiertes Vertrauen in andere Menschen. Denn Einschätzungen bezüglich der Vertrauenswürdigkeit fremder Menschen speisen sich aus Generalisierungen früherer Erfahrungen (Hardin 1996: 27). Vertrauen wird somit in konkreten positiven Interaktionserfahrungen im Rahmen von Vereinsaktivitäten generiert und anschliessend auf die breitere Gesellschaft ausserhalb dieser Vereinigungen generalisiert (Caulkins 2004: 164). Aus diesem Grund wird auch vor allem aktiver Partizipation und nicht der blossen Mitgliedschaft eine vertrauensfördernde Wirkung zugeschrieben, da nur bei ersterer die entscheidende direkte Interaktion mit anderen Mitgliedern stattfindet (Newton 2004: 20; Wollebæk/Selle 2003: 68).

Zusammengefasst sollten die Vermittlung von gesellschaftlichen Werten, die Regelung gemeinschaftlicher Interaktionen sowie das Sammeln positiver Erfahrungen mit Mitmenschen in einem starken Zusammenhang zwischen der aktiven zivilgesellschaftlichen Partizipation und dem zwischenmenschlichen Vertrauen resultieren. Bisherige theoretische Ansätze sowie empirische Befunde lassen allerdings vermuten, dass sich nicht alle Arten zivilgesellschaftlicher Vereinigungen in diesem Effekt auf die Ausbildung von Vertrauen gleichen (Hooghe/Stolle 2003: 10f.; Howard/Gilbert 2008; Letki 2004: 667; Kwak et al. 2004; Paxton 2007; Stolle 2003: 25f.; Stolle/Rochon 1998; Wollebæk/Selle 2003; Zmerli 2003). Vielmehr sollten manche Typen von Vereinen und Organisationen aufgrund bestimmter Charakteristika eher zur Ausbildung von Vertrauen beitragen als andere. Die Forschung unterscheidet hier zwischen brückenbildenden und abgrenzenden Vereinigungen einerseits sowie verbundenen und isolierten Gruppen andererseits. Während diese Perspektiven den Vereinstypus in den Mittelpunkt ihres analytischen Interesses stellen, rückt das später $\mathrm{zu}$ präsentierende Konzept der vereinstypusunabhängigen individuellen Mehrfachmitgliedschaften allein den Grad der persönlichen Einbindung in eine undifferenzierte Vereinswelt in den Vordergrund. 


\section{Brückenbildende und abgrenzende Vereinigungen}

Um diese vertrauensrelevanten Charakteristiken genauer zu begründen, muss zunächst zwischen brückenbildenden ("bridging") und abgrenzenden ("bonding") Vereinen unterschieden werden (Putnam 2000; Putnam/Goss 2001). Letztere bezeichnen Netzwerke, welche eher nach innen auf die Erringung von Vorteilen der eigenen Klientel ausgerichtet sind und deren Mitglieder sich durch eine ausgeprägte Homogenität bezüglich bestimmter sozialer Eigenschaften und Merkmale auszeichnen (vgl. Zmerli 2003: 68). Solche Netzwerke fördern aufgrund gemeinsamer Identifikation einen starken Zusammenhalt innerhalb der Gruppe, weisen jedoch nur eine geringe Verknüpfung zur restlichen Gesellschaft auf. Bei brückenbildenden Organisationen ist es nach Putnam (2000: 411) hingegen von Bedeutung, dass soziale, professionelle, religiöse oder auch politische Identitäten transzendiert werden. Gemeint sind folglich soziale Netzwerke, "die völlig unterschiedliche Menschen zusammenbringen" (Putnam/Goss 2001: 28). Diese sind eher über die eigenen Organisationsgrenzen hinaus orientiert und schaffen darüber breitere Identitäten und gesellschaftliche Werte (Putnam 2000: 22f.).

Innerhalb der Sozialkapitalforschung wird deshalb gemeinhin angenommen, dass brückenbildenden Netzwerkén im Vergleich zu abgrenzenden Netzwerken hinsichtlich der Generierung Allgemeinwohl fördernder Externalitäten eine bedeutendere Rolle zukommt. Brückenbildende Organisationen und Vereine sollten durch ihre Aussenorientierung und das damit verbundene Schaffen erweiterter Identitäten positive Einstellungen bezüglich der Kooperation mit anderen Mitgliedern der Gesellschaft fördern (Caulkins 2004: 165; Putnam 2000: 23). Darüber hinaus ermöglicht die heterogene Mitgliederstruktur solcher Netzwerke Interaktionen zwischen Menschen, die unterschiedlich sind. Die daraus resultierenden Erfahrungen zur Vertrauenswürdigkeit anderer können in der Folge leichter auf eine heterogene Gesellschaft projiziert und generalisiert werden (Zmerli 2003):

[P]ositive experiences with dissimilar individuals will have greater effects on the development of generalized trust than will the relations with individuals who are similar to oneself in terms of their characteristics, attitudes or behaviors (Marshall/ Stolle 2004: 129).

Im Vergleich dazu schaffen nach Putnam (2000: 23) abgrenzende Organisationen durch ihre grosse Homogenität sowie Innenorientierung eher eine starke Loyalität gegenüber der eigenen Gruppe, welche jedoch gleichzeitig 
eine Abschottung nach aussen zur Folge haben könnte (vgl. Zmerli 2003: 68). Menschen, welche innerhalb solcher Netzwerke miteinander direkt interagieren, werden zwar dazu neigen, starkes Vertrauen in die anderen Mitglieder auszubilden. Generalisiertes Vertrauen in Menschen jenseits der eigenen Gruppe sollte jedoch nicht gefördert werden. Möglicherweise entsteht sogar eher Misstrauen in andere Menschen im Allgemeinen. Diese Überlegungen lassen sich in folgender Hypothese zusammenfassen:

HI: Personen, welche in brückenbildenden Organisationen aktiv tätig sind, sollten ein grösseres generalisiertes Vertrauen in andere Menschen aufweisen. Die aktive Mitgliedschaft in abgrenzenden Organisationen, welche in sich homogen und nach innen orientiert sind, fördert hingegen kein Vertrauen gegenüber den Mitmenschen im Allgemeinen.

\section{Verbundene und isolierte Vereinigungen}

Eine zweite, vereinsdifferenzierende Anschauung stellt das Merkmal der Verbundenheit oder Isolation von Organisationen in den Mittelpunkt des analytischen Interesses (Paxton 2002, 2007). Im Gegensatz zur Unterscheidung von brückenbildenden und abgrenzenden Organisationen, welche auf die Struktur innerhalb von einzelnen Vereinen abzielt, fokussiert diese Perspektive jedoch auf die Struktur zwischen den Vereinen. Eine Organisation gilt dann als besonders verknüpft oder verbunden, wenn sie über ihre jeweiligen Mitglieder eine Vielzahl an Verbindungen oder Anknüpfungspunkten zu anderen Vereinen aufweisen kann. Verbundene Vereinigungen sollten die Ausbildung generalisierten Vertrauens stärker als isolierte Vereine fördern, da sie dazu beitragen, soziale Netzwerke über den engen Rahmen einer einzigen Organisation hinaus zu spannen (Paxton 2007: 51). Diese durch einzelne Mitglieder hergestellte Verbindung zwischen verschiedenen Vereinigungen erleichtert die Ausweitung des Vertrauensradius, da auf diese Weise Bekanntschaft und Vertrautheit zwischen Menschen geschaffen werden, die selbst nicht unmittelbare Interaktionspartner sind. Nichtmitglieder einer Organisation werden eher als vertrauenswürdig eingestuft, wenn bekannte Vereinsmitglieder mit eben diesen Nichtmitgliedern in anderen gemeinsamen Organisationskontexten in direktem Kontakt stehen. So wird eine Person einer anderen eher vertrauen, wenn sie weiss, dass ein Mitglied der eigenen Organisation mit dieser regelmässig in einer anderen Vereinigung kooperiert (ebd.). 
Darüberhinaus erhöht die Zahl der Verbindungen auch den Grad der Diversität der Kontakte und fördert auch auf diesem Wege das generalisierte Vertrauen. So konstatieren Wollebæk/Selle (2003: 70), "the more overlapping and interlocking networks that exist, the higher the probability that people from different backgrounds will meet". Organisationen, welche durch einen hohen Anteil an solchen Mehrfachmitgliedern eng mit anderen Organisationen und Vereinen verbunden sind, generieren über die daraus entstehenden Verknüpfungen breitere Identitäten und sind weniger auf abgrenzende Gruppencharakteristiken fixiert (Paxton 2007: 51). Dadurch sollte sich das Vertrauen der Mitglieder leichter auf die gesamte Gesellschaft verallgemeinern lassen. Paxton (2007) identifiziert beispielsweise religiöse Vereinigungen, Sportvereine und Gewerkschaften als isolierte Gruppen, da deren Mitglieder vergleichsweise wenige Verknüpfungen zu anderen Vereinen aufweisen. Tierschutz- und Menschenrechtsvereinen wie auch Umweltbewegungen wird hingegen ein hohes Verbindungspotential und damit die Rolle von besonderen Vertrauensgeneratoren zugeschrieben. Daraus lässt sich schliesslich die folgende Hypothese ableiten:

H2: Aktive Mitglieder verbundener Organisationen, die über Mehrfachmitglieder vielfältig mit unterschiedlichen Vereinstypen verknüpft sind, sollten im Vergleich zu aktiven Mitgliedern isolierter Vereine und Organisationen ein grösseres generalisiertes Vertrauen aufweisen.

\section{Individuelle vereinstypusunabhängige Mehrfachmitgliedschaften}

Das analytische Potential der beiden erwähnten Ansätze besteht zum einen in der differenzierten Betrachtung der Vereinslandschaft, die ihrer empirischen Vielfalt Rechnung trägt. Zum anderen präzisieren sie die theoretischen Wirkungsmechanismen, durch welche generalisiertes Vertrauen in Vereinen generiert wird. Dennoch vermag ihre Erklärungskraft bislang nicht vollständig zu überzeugen (Coffé/Geys 2007; Uslaner 2002). So sind etwa die meisten Assoziationen in der Praxis brückenbildend und abgrenzend zugleich (Putnam/Goss 2001: 29). Beispielsweise kann eine Gruppe Personen umfassen, die verschiedenen sozioökonomischen Schichten oder Volksgruppen (brückenbildende Merkmale) angehören und gleichzeitig dasselbe Geschlecht (abgrenzendes Merkmal) aufweisen (beispielsweise homosexuelle Vereinigungen). Gegenüber der Unterscheidung zwischen isolierten und verbundenen Assoziationen wiederum wird eingebracht, 
diese Anschauung vernachlässige den Aspekt der Gruppengrösse: Da mitgliederstarke Vereinigungen generell höhere Verbindungen $\mathrm{zu}$ anderen Vereinen aufweisen, sei das Ausmass möglicher Verknüpfungen einzig eine Funktion der Grösse der jeweiligen Organisation (Coffé/Geys 2007: 125).

Im Gegensatz zu den bislang genannten Perspektiven setzt die Sichtweise der individuellen vereinstypusunabhängigen Mehrfachmitgliedschaften weniger an der Meso- als vielmehr an der Mikroebene der Individuen an. Während die Ansätze zu den brückenbildenden und abgrenzenden Vereinigungen einerseits, sowie zu den verbundenen und isolierten Gruppen andererseits, auf die Beschaffenheit der einzelnen Vereine fokussieren, stellt der Zugang der individuellen vereinstypusunabhängigen Mehrfachmitgliedschaften die Prägekraft bestimmter Vereinstypen in Frage und fokussiert auf die Einbindung der Individuen jenseits theoretisch wie empirisch abgeleiteter Vereinsdifferenzierungen. Mit anderen Worten: Während etwa die Forschungen von Paxton (2007) und Zmerli (2003) anhand theoretischer wie empirischer Erwägungen die Vereine unterteilen und von der zentralen Annahme geleitet werden, dass nicht alle Typen von Freiwilligenorganisationen in Bezug auf die Vertrauensbildung als gleich zu betrachten sind, verlegt der Ansatz der persönlichen vereinstypusunabhängigen Mehrfachmitgliedschaften die Perspektive auf die Ähnlichkeit von Vereinen. Entscheidend ist hier allein die Breite, sprich die bloße Anzahl aktiver zivilgesellschaftlicher Tätigkeiten - ganz unabhängig vom konkreten Vereinstypus.

Folgt man der Logik, wonach ein breiter positiver Erfahrungsschatz zur Generalisierung von Vertrauen führt (Hardin 1996), sollten insbesondere diejenigen Individuen großes Vertrauen in die Mitmenschen haben, welche Mitgliedschaften in einer Vielzahl von Organisationstypen aufweisen. Dieser Effekt sollte dabei unabhängig davon auftreten, in welchen konkreten Typen von Netzwerken die betreffende Person partizipiert. Dies bedeutet, selbst wenn man sich in Freiwilligenorganisationen engagiert, die in sich sehr homogen sind und nur über wenige überlappende Mitgliedschaften mit anderen Organisationen verfügen, sollte der Aspekt der Interaktion in mehreren verschiedenen Organisationstypen zur Folge haben, dass man mit unterschiedlicheren Menschen interagiert, als wenn man nur in einer dieser Organisationen tätig wäre. Dies führt zu einem breiteren Erfahrungsschatz bezüglich der Kooperation mit verschiedensten Individuen und deren $\mathrm{Zu}$ verlässigkeit. Nach Wollebæk/Selle (2003: 70), "multiple affiliations mean more and broader interaction. Consequently, it should have a cumulative 
effect on an individual's trust"'. Diese Überlegungen führen zu folgender Hypothese:

H3: Personen, die in mehreren Vereinen und Organisationen aktiv partizipieren, sollten mehr Vertrauen in andere besitzen als Individuen, welche nur in einer oder überhaupt keiner Freiwilligenorganisation engagiert sind. Dem Vereinstypus kommt hierbei keine entscheidende Bedeutung zu.

\section{Die Frage der Kausalität}

Ein für die hier behandelte Thematik wichtiger Aspekt betrifft zuletzt noch die Frage nach der Kausalität der postulierten Zusammenhänge. Alle der bisher vorgebrachten Argumente zum Verhältnis von aktivem Engagement in zivilgesellschaftlichen Vereinigungen und dem generalisierten Vertrauen gehen davon aus, dass die kausale Richtung des Wirkungsmechanismus tatsächlich von den Vereinigungen hin zum Vertrauen verläuft. Diese Annahme ist jedoch keineswegs unwidersprochen geblieben (Stolle 1998; Uslaner 2002). In der Tat lassen sich auch theoretische Gründe für ein umgekehrtes Kausalverhältnis anführen. So könnten etwa besonders vertrauensvoll eingestellte Personen eher die Bereitschaft aufweisen, sich überhaupt in zivilgesellschaftlichen Gruppen zu engagieren und dort mit Menschen aus unterschiedlichen Kontexten zu kooperieren.

Im Rahmen der vorliegenden Studie kann diese Frage nicht abschlieBend geklärt werden - dazu wären vielmehr Paneldaten vonnöten, welche die aktiven Mitgliedschaften und das generalisierte Vertrauen der Befragten über einen längeren Zeitraum erfassen. ${ }^{4}$ Gleichwohl wird im Großteil der theoretischen Literatur davon ausgegangen, dass "causation flows mainly from joining to trusting" (Putnam 1995: 666), und auch eine Reihe von empirischen Studien unterstützen diesen Standpunkt. In ihrer Analyse für die USA kommen etwa Brehm und Rahn (1997) zu dem Schluss, der Effekt von Vereinsmitgliedschaft auf Vertrauen sei größer als jener von Vertrauen auf Vereinsmitgliedschaft. Claibourn und Martin (2000) betrachten den Zusammenhang in Längsschnittsperspektive und können keinen Einfluss

\footnotetext{
4 Im Schweizer Haushalts-Panel (SHP) etwa sind diese Informationen nur für den kurzen Zeitraum von 2002 bis 2006 enthalten. Erste, auf diesem beschränkten Datenmaterial basierende Analysen weisen auf ein wechselseitiges Bedingungsverhältnis hin, wobei sich jedoch der Effekt von Vereinsmitgliedschaft auf Vertrauen als etwas stärker herausstellt, als umgekehrt.
} 
von Vertrauen auf Vereinsmitgliedschaft feststellen, wohl aber einen Effekt des Engagements auf das Vertrauen. Shah (1998) schließlich modelliert ein reziprokes Kausalverhältnis zwischen beiden Größen und resümiert, dass nur Vereinsaktivität auf generalisiertes Vertrauen wirkt und nicht umgekehrt. Alles in allem erscheint es uns daher gerechtfertigt, von der vorgeschlagenen Kausalrichtung auszugehen, auch wenn wir diese aufgrund des gegebenen Datenmaterials selbst nicht unmittelbar überprüfen können.

\section{Daten und Methoden}

Die Grundlage der empirischen Analyse bilden die Daten des Schweizer Freiwilligen-Monitors 2007, in dessen Rahmen 7'410 Personen zu verschiedenen Formen und Erfahrungen freiwilliger Tätigkeit befragt wurden. ${ }^{5}$ Der Freiwilligen-Monitor bietet daher die Möglichkeit, die Beziehung zwischen aktiver Tätigkeit in Freiwilligenorganisationen und dem generalisierten Vertrauen empirisch anhand einer breiten Datenbasis für die Schweiz zu analysieren.

Die abhängige Variable, das generalisierte Vertrauen in andere Menschen, wird analog zu annähernd allen einschlägigen Studien in diesem Forschungsbereich über die Frage "Würden Sie sagen, dass man den meisten Menschen vertrauen kann, oder kann man im Umgang mit anderen Menschen nicht vorsichtig genug sein? " erhoben (siehe z.B. Delhey/Newton 2002; Freitag 2003; Uslaner 2002). Im Freiwilligen-Monitor wird dabei eine Antwortskala vorgegeben, welche Werte von 0 bis 10 umfasst, wobei 10 bedeutet, dass man den meisten Menschen vertrauen kann und 0 , dass man im Umgang mit anderen nicht vorsichtig genug sein kann. Diese Variable wird im Folgenden als quasi-metrisch skaliert behandelt, um sie im Rahmen einfacher linearer Regressionen (OLS) zu modellieren. Diesem Verfahren wurde gegenüber einer Ordered Logit Regression

\footnotetext{
5 Beim Freiwilligen-Monitor handelt es sich um eine Initiative unter der Trägerschaft der Schweizerischen Gemeinnützigen Gesellschaft, dem Migros-Kulturprozent und dem Bundesamt für Statistik, welche das Ziel verfolgt, das freiwillige Engagement in der Schweiz in differenzierter Weise zu beleuchten (Stadelmann-Steffen et al. 2007). Im Zuge dessen wurde der Freiwilligen-Monitor im Jahre 2006 erstmals über eine repräsentative Telefonumfrage der Schweizer Bevölkerung über 15 Jahre erhoben $\left(\mathrm{N}=7^{\prime} 410\right)$. Die Auswahl der Befragten erfolgte anhand einer mehrstufigen Zufallsstichprobe der Haushalte sowie der Person innerhalb des jeweiligen Haushalts. Um für jeden Kanton eine Mindestanzahl an Befragten verwirklichen zu können, wurde die Stichprobe dabei disproportional geschichtet gezogen. Sämtliche Materialien zur Piloterhebung des "Schweizerischen FreiwilligenMonitors" sind unter http://www.freiwilligen-monitor.ch einzusehen.
} 
für ordinal skalierte Variablen vor allem deshalb der Vorzug gegeben, da es eine deutlich intuitivere Interpretation der Koeffizienten zulässt. Die Ergebnisse beider Verfahren unterscheiden sich freilich nicht substantiell voneinander.

Um die zentrale unabhängige Variable, das aktive Engagement in Freiwilligenorganisationen, $\mathrm{zu}$ messen, wurde für zehn vorgegebene Organisationstypen und Tätigkeitsfelder separat erhoben, ob die befragte Person innerhalb der vergangenen 12 Monate in einem solchen Organisationstyp aktiv tätig war. ${ }^{6}$ Zur Erfassung der aktiven Tätigkeit in formellen Freiwilligenorganisationen allgemein wird eine dichotome Variable generiert. Als aktiv engagiert gelten dabei diejenigen Personen, welche in mindestens einem der vorgegebenen Tätigkeitsfelder aktiv tätig waren bzw. sind. Nach den Zahlen des Freiwilligen-Monitors sind dies immerhin 57 Prozent der Schweizer Bevölkerung.

Um zwischen "brückenbildenden" und "abgrenzenden" Vereinigungen unterscheiden zu können, wurden für die zehn verschiedenen vorgegebenen Organisationstypen in Anlehnung an Coffé und Geys (2007) normalisierte Diversity Scores berechnet. ${ }^{7}$ Diese Masszahl berechnet sich aus der absoluten Abweichung der sozialen Komposition eines Vereinstyps von der tatsächlichen Merkmalsverteilung in der Gesamtbevölkerung. ${ }^{8}$ Je stärker

\footnotetext{
" Der genaue Wortlaut der Frage ist folgender: "Es gibt verschiedene Möglichkeiten, sich ausserhalb von Beruf und Familie irgendwo zu engagieren, beispielsweise in einem Verein oder einer Organisation. Ich nenne Ihnen nun verschiedene Möglichkeiten, bitte sagen Sie mir anhand der Antwortvorgaben, ob einer der Punkte auf Sie zutrifft. Uns interessiert, ob Sie in diesen Organisationen in den letzten 12 Monaten Mitglied sind/waren, etwas gespendet haben oder aktiv mitmachen/mitgemacht haben oder ob davon nichts auf Sie zutrifft".

${ }^{7}$ Einen alternativen Vorschlag zur Erfassung des brückenbildenden oder abgrenzenden Charakters von Vereinigungen auf Basis ihrer konstitutiven Güter hat Zmerli $(2003,2008)$ vorgebracht.

${ }^{8}$ Der Diversity Score eines Vereinstyps $i$ hinsichtlich des sozialen Merkmals $x$ mit $k$ Merkmalsausprägungen ergibt sich aus

$$
\text { Diversity Score }_{i x}=\left(\sum_{k=1}^{n}\left|a_{k \text { Gesamtpopulation }}-a_{k i}\right|\right) / n
$$

wobei $a_{k}$ die Anteile der $k$ ten $(1 \ldots n)$ Merkmalsausprägung darstellen. Die auf diese Weise für verschiedene sozial relevante Merkmale $(x)$ berechneten Scores wurden über alle Vereinstypen $(i)$ hinweg zwischen 0 und 1 normalisiert und schliesslich für jeden Vereinstyp zu einem Gesamtmass aufaddiert (vgl. Coffé/Geys 2007: 126). Als sozial relevante Merkmale wurden in der Berechnung Geschlecht, Alter, Staatsangehörigkeit, Religionszugehörigkeit, Bildung, Haushaltseinkommen sowie der Erwerbsstatus berücksichtigt.
} 
Tabelle 1: Brückenbildende und abgrenzende Vereinigungen in der Schweiz

\begin{tabular}{lcc}
\hline Organisationstyp & $\begin{array}{c}\text { Summe der } \\
\text { normalisierten } \\
\text { Diversity Scores }^{\mathrm{a}}\end{array}$ & $\begin{array}{c}\text { Bevölkerungs- } \\
\text { anteil in Prozent }\end{array}$ \\
\hline Abgrenzende Vereinigungen & & \\
Politische oder öffentliche Ämter & 5.64 & 4.0 \\
Politische Parteien & 4.18 & 3.6 \\
Interessenverbände & 3.35 & 7.5 \\
Öffentliche Dienste & 3.14 & 7.4 \\
Brückenbildende Vereinigungen & & \\
Menschenrechte/Umweltverbände & 2.44 & 3.2 \\
Soziale/karitative/gemeinnützige Institutionen & 2.42 & 6.6 \\
Spiel-/Hobby-/Freizeitvereine & 2.20 & 19.6 \\
Sportclubs, -vereine & 1.85 & 28.1 \\
Kulturelle Vereine & 1.84 & 12.6 \\
Kirche oder kirchennahe Organisationen & 1.49 & 13.7 \\
\hline
\end{tabular}

Anmerkungen: Gewichtete Angaben; N = 7\%410; ${ }^{a}$ berechnet nach Coffé/Geys (2007).

die Abweichung hinsichtlich eines sozial relevanten Merkmals, desto stärker sind Menschen mit diesem Merkmal innerhalb eines Organisationstyps über- oder unterrepräsentiert und desto geringer folglich die Wahrscheinlichkeit brückenbildender Kontakte. Geringe Index-Werte deuten dabei auf ein hohes brückenbildendes Potential des Vereinigungstyps hin. Tabelle 1 zeigt die berechneten Werte sowie die darauf beruhende Einteilung verschiedener Vereinstypen als brückenbildende oder abgrenzende. ${ }^{9}$

Politische und öffentliche Ämter, politische Parteien, Interessensverbände sowie öffentliche Dienste zählen demnach zu eher abgrenzenden zivilgesellschaftlichen Organisationsformen. Vereinigungen mit brückenbildendem Charakter stellen demgegenüber Kirchen, Kultur-, Sport- und Freizeitvereine, sowie soziale Organisationen und Umweltverbände dar. Aus dieser Einteilung wurden zwei Dummy-Variablen generiert, die einerseits aktives Engagement in mindestens einer abgrenzenden und andererseits aktive Partizipation in mindestens einer brückenbildenden Verei-

9 Da die hier angewandte Methode den Unterschied zwischen brückenbildenden und abgrenzenden Vereinigungen als einen graduellen erfasst, liegt die konkrete Einteilung der verschiedenen Vereinstypen in die eine oder andere Kategorie im Ermessensspielraum des Forschers (vgl. Coffé/Geys 2007: 128). 
Tabelle 2: Isolierte und verbundene Vereinigungen in der Schweiz

\begin{tabular}{lcc}
\hline Organisationstyp & $\begin{array}{c}\text { aktive Mehrfach- } \\
\text { mitglieder } \\
\text { in Prozent }\end{array}$ & $\begin{array}{c}\text { durchschnittliche } \\
\text { Zahl an } \\
\text { Mitgliedschaften }\end{array}$ \\
\hline Isolierte Vereinigungen & 63.1 & 2.1 \\
Sportclubs, -vereine & 66.6 & 2.4 \\
Kirche oder kirchennahe Organisationen & & \\
Verbundene Vereinigungen & 75.8 & 2.7 \\
Interessenverbände & 78.8 & 2.7 \\
Soziale/karitative/gemeinnützige Institutionen & 79.6 & 2.6 \\
Kulturelle Vereine & 79.4 & 2.4 \\
Spiel-/Hobby-/Freizeitvereine & 81.9 & 2.8 \\
Öffentliche Dienste & 84.4 & 3.0 \\
Menschenrechte/Umweltverbände & 87.2 & 3.2 \\
Politische Parteien & 92.1 & 3.4 \\
Politische oder öffentliche Ämter & & \\
\hline
\end{tabular}

Anmerkungen: Gewichtete Angaben; N = 7’410; vgl. Paxton 2007.

nigung anzeigen. Dabei sind etwa 18 Prozent der Schweizer Bevölkerung in mindestens einer abgrenzenden und 53 Prozent in mindestens einer brückenbildenden Organisation engagiert.

Zusätzlich sollen analog zu Paxton (2007) Organisationstypen mit verhältnismässig wenigen Verknüpfungen als isolierte, zahlreich verknüpfte Freiwilligenorganisationen hingegen als verbundene Organisationen betrachtet werden. Dazu wurden für jeden Vereinstyp einerseits die Anteile der Mehrfachmitglieder sowie die durchschnittliche Anzahl der Mitgliedschaften ihrer Mitglieder berechnet. Isolierte Vereinigungstypen sind demnach jene, deren Mitglieder kaum Mehrfachmitgliedschaften und damit kaum Verknüpfungen zu weiteren Vereinen aufweisen (siehe Tabelle 2). So scheinen sowohl Sportvereine als auch kirchliche Organisationen über weitaus weniger überlappende aktive Mitgliedschaften zu anderen Tätigkeitsfeldern von Freiwilligenorganisationen zu verfügen, weshalb sie im Folgenden analog zu Paxton (2007) zum Typ der isolierten Organisationen gezählt werden. Die restlichen Organisationstypen scheinen hingegen über hohe Anteile an Mehrfachmitgliedern Verbindungen zur breiteren Gesellschaft aufgebaut zu haben. Die aktive Tätigkeit in mindestens einer isolierten bzw. verbundenen Organisation wird wiederum als Dummy-Va- 
riable codiert. Etwa 38 Prozent der Schweizer Bevölkerung sind in mindestens einer isolierten und 41 Prozent in mindestens einer verbundenen Organisation engagiert.

Zur Erfassung der Intensität individueller Interaktionen mit verschiedenen Menschen über die vereinstypusunabhängigen Mehrfachmitgliedschaften wird zuletzt eine Variable gebildet, welche die Anzahl der aktiven Mitgliedschaften in unterschiedlichen Vereins- und Organisationstypen wiedergibt. Während 27 Prozent der Schweizer lediglich in einem Organisationstyp engagiert sind, sind weitere 30 Prozent Mehrfachmitglieder.

Um der Gefahr möglicher Scheinkorrelationen, Suppressionen oder Verzerrungen vorzubeugen, werden neben den aufgeführten Indikatoren auch Kontrollvariablen, welche potenziell Einfluss auf das generalisierte Vertrauen ausüben sollten, in der Analyse berücksichtigt. ${ }^{10}$ Dies umfasst zunächst die naheliegenden soziodemographischen Charakteristiken, wie das Geschlecht, das Alter und den persönlichen Bildungsgrad, gemessen über den höchsten erworbenen Bildungsabschluss. Ergänzend wurde in der Vergangenheit des Öfteren argumentiert, dass generalisiertes Vertrauen in erster Linie bei den Gewinnern der Gesellschaft zum Ausdruck kommt und stark mit dem Vorhandensein persönlicher Ressourcen zusammenhängt (Freitag 2003; Putnam 2000). Dieser Aspekt des Erfolgs und der Zufriedenheit im Leben soll zum einen durch die subjektive Einschätzung der eigenen Lebenszufriedenheit berücksichtigt werden. Zum anderen wird die Verfügbarkeit persönlicher Ressourcen über das monatlich verfügbare Haushaltseinkommen in die Untersuchung einbezogen. Ausländer könnten aufgrund ihrer Herkunftskultur oder aufgrund von Diskriminierungserfahrungen ein geringeres Vertrauen in die Mehrheit aufweisen. Des Weiteren ist zu erwarten, dass Individuen anderen Menschen vertrauen, solange sie in ihren politischen Institutionen Apparate sehen, welche vertrauenswürdiges Verhalten fördern und opportunistisches Verhalten wie Lügen, Stehlen oder Betrügen sanktionieren oder bestrafen (Freitag/Bühlmann 2009; Stolle/Rothstein 2007). Das Vertrauen in politische Institutionen wird in dieser Studie über die Zufriedenheit mit dem Funktionieren der Demokratie im eigenen Land erhoben. Zusätzlich stellen religiöse Kulturwerte eine entscheidende Grundlage sozialen Vertrauens dar (Fukuyama 1995; Traunmüller 2009). Im Folgenden sollen diese über die jeweilige Konfessionszugehörigkeit bestimmt werden. Als Alternative zum freiwilligen En-

${ }^{10}$ Eine detaillierte Beschreibung aller verwendeten Variablen befindet sich in Tabelle Al im Anhang. 
gagement in formellen Organisationen können letztlich informelle Netzwerke gesehen werden, die ebenfalls die Möglichkeit bieten, Erfahrungen bezüglich der Vertrauenswürdigkeit anderer zu machen und Verhaltensnormen zum Umgang miteinander zu verinnerlichen. Als Indikator für die Teilnahme an solchen informellen Netzwerken dient hier die Häufigkeit des Treffens mit Freunden, Verwandten oder Arbeitskollegen ausserhalb des Berufslebens.

Während für sämtliche deskriptive Statistiken eine Gewichtung berücksichtigt wurde, findet diese in den Regressionsmodellen keine Verwendung. In den Modellen wurden nur Fälle ohne fehlende Variablenwerte berücksichtigt.

\section{Empirische Befunde}

Im Folgenden soll analysiert werden, in welchem Zusammenhang aktives Vereinsengagement mit generalisiertem Vertrauen steht. Tabelle 3 zeigt zunächst die Ergebnisse einer multiplen Regression der aktiven Mitgliedschaft in Freiwilligenorganisationen auf das Vertrauen unter Einbezug verschiedener Kontrollvariablen (Modell 1). Blickt man auf die aktive Tätigkeit in Freiwilligenorganisationen als primär interessierende Einflussgrösse, so offenbart sich ein positiver Zusammenhang zwischen aktiver Mitgliedschaft und Vertrauen. Menschen, die in einer zivilgesellschaftlichen Vereinigung aktiv sind, sind in der Tat eher bereit, ihren Mitmenschen im Allgemeinen zu vertrauen.

Die zentrale Frage dieses Beitrags ist jedoch, ob alle Typen von Freiwilligenorganisationen hinsichtlich dieser Beziehung als gleichwertig zu betrachten sind. Es ist deshalb von Bedeutung, in der weiteren Analyse zwischen verschiedenen Arten von Organisationen zu unterscheiden. Eine getrennte Betrachtung der aktiven Tätigkeit in brückenbildenden bzw. abgrenzenden Organisationen gibt dabei jedoch nur wenig Aufschluss (Modell 2). So zeigt sich die Verknüpfung von Vertrauen und der aktiven Partizipation in abgrenzenden oder brückenschlagenden Organisationen nicht in der erwarteten Art und Weise. Die These, dass Menschen grösseres Vertrauen in andere aufweisen, wenn sie in heterogenen, nach aussen orientierten Netzwerken aktiv partizipieren, findet zwar Unterstützung. Doch dasselbe lässt sich für die Partizipation in abgrenzenden Vereinstypen sagen. Tatsächlich unterscheidet sich der Effekt der beiden Vereinigungsformen auch nicht voneinander $\left(\chi^{2}\left(1,6^{\prime} 194\right)=0.21, p=0.65\right)$. Die pos- 
Tabelle 3: Regressionsmodelle für generalisiertes Vertrauen I (OLS)

\begin{tabular}{|c|c|c|c|c|c|c|}
\hline \multirow[b]{2}{*}{ Aktives Vereinsengagement } & \multicolumn{2}{|c|}{ M1 } & \multicolumn{2}{|c|}{ M2 } & \multicolumn{2}{|c|}{ M3 } \\
\hline & $0.19 * * *$ & $(0.06)$ & & & & \\
\hline Abgrenzende Vereinigung & & & $0.20 * *$ & $(0.07)$ & & \\
\hline $\begin{array}{l}\text { Brückenbildende } \\
\text { Vereinigung }\end{array}$ & & & $0.16^{* *}$ & $(0.06)$ & & \\
\hline Isolierte Vereinigung & & & & & $0.13^{*}$ & $(0.06)$ \\
\hline Verbundene Vereinigung & & & & & $0.19 * * *$ & $(0.06)$ \\
\hline Männlich & $-0.17^{* *}$ & $(0.05)$ & $-0.19 * * *$ & $(0.06)$ & $-0.18 * * *$ & $(0.05)$ \\
\hline Alter & $0.01^{* * *}$ & $(0.00)$ & $0.01 * * *$ & $(0.00)$ & $0.01 * * *$ & $(0.00)$ \\
\hline Ausländer & $-0.26 * *$ & $(0.08)$ & $-0.24 * *$ & $(0.08)$ & $-0.23 * *$ & $(0.08)$ \\
\hline Protestantisch ${ }^{\mathrm{a}}$ & 0.01 & $(0.08)$ & 0.00 & $(0.08)$ & -0.00 & $(0.08)$ \\
\hline Katholischa & -0.11 & $(0.08)$ & -0.11 & $(0.08)$ & -0.12 & $(0.08)$ \\
\hline Andere Christl. ${ }^{a}$ & $-0.43 * * *$ & $(0.13)$ & $-0.43^{* * *}$ & $(0.13)$ & $-0.44 * * *$ & $(0.13)$ \\
\hline Andere Nicht-christl." & $-0.45^{* *}$ & $(0.15)$ & $-0.45^{* *}$ & $(0.15)$ & $-0.46^{* *}$ & $(0.15)$ \\
\hline Bildung: Sekundärstufe I ${ }^{\mathrm{b}}$ & -0.26 & $(0.17)$ & -0.26 & $(0.17)$ & -0.26 & $(0.17)$ \\
\hline Bildung: Sekundärstufe II $^{\mathrm{b}}$ & 0.10 & $(0.16)$ & 0.09 & $(0.16)$ & 0.09 & $(0.16)$ \\
\hline Bildung: Tertiär ${ }^{b}$ & $0.42 *$ & $(0.17)$ & $0.41^{*}$ & $(0.17)$ & $0.41^{*}$ & $(0.17)$ \\
\hline Mittleres Einkommen ${ }^{c}$ & $0.18^{* *}$ & $(0.06)$ & $0.17 * *$ & $(0.06)$ & $0.17 * *$ & $(0.06)$ \\
\hline Hohes Einkommen ${ }^{c}$ & 0.16 & $(0.12)$ & 0.15 & $(0.12)$ & 0.15 & $(0.12)$ \\
\hline $\begin{array}{l}\text { Einkommensangabe } \\
\text { verweigert }^{c}\end{array}$ & $-0.27 * *$ & $(0.09)$ & $-0.27 * *$ & $(0.09)$ & $-0.28 * *$ & $(0.09)$ \\
\hline Lebenszufriedenheit & $0.20 * * *$ & $(0.02)$ & $0.20 * * *$ & $(0.02)$ & $0.20 * * *$ & $(0.02)$ \\
\hline Demokratiezufriedenheit & $0.17^{* * *}$ & $(0.01)$ & $0.17 * * *$ & $(0.01)$ & $0.17 * * *$ & $(0.01)$ \\
\hline $\begin{array}{l}\text { Treffen mit Freunden, etc.: } \\
\text { wenige Male im Monat }{ }^{d}\end{array}$ & $0.99 * * *$ & $(0.14)$ & $0.98 * * *$ & $(0.14)$ & $0.97^{* * *}$ & $(0.14)$ \\
\hline $\begin{array}{l}\text { Treffen mit Freunden: } \\
\text { mehrmals pro Woche }\end{array}$ & $1.28 * * *$ & $(0.14)$ & $1.27 * * *$ & $(0.14)$ & $1.26^{* * *}$ & $(0.14)$ \\
\hline Konstante & $2.29 * * *$ & $(0.26)$ & $2.32 * * *$ & $(0.26)$ & $2.31 * * *$ & $(0.26)$ \\
\hline F-Statistik & 43.395 & $* * * *$ & 41.590 & $* * *$ & 41.74 & $* * *$ \\
\hline Adj. $R^{2}$ & 0.1 & & 0.1 & & 0.1 & \\
\hline $\mathrm{N}$ & $6^{\prime} 21$ & & $6: 21$ & & $6: 2$ & \\
\hline
\end{tabular}

Anmerkungen: Unstandardisierte Regressionskoeffizienten, Standardfehler in Klammern; ${ }^{*} \mathrm{p}<0.05,{ }^{* *} \mathrm{p}<0.01,{ }^{* * *} \mathrm{p}<0.001$. ${ }^{\text {a }}$ Referenzkategorie: konfessionslos; ${ }^{b}$ Referenzkategorie: kein vollendeter Bildungsabschluss; ${ }^{\circ}$ Referenzkategorie: niedriges Einkommen

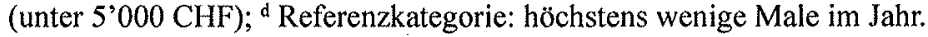


tulierte Überlegenheit brückenbildender im Gegensatz zu abgrenzenden Freiwilligenorganisationen hinsichtlich der Vertrauensbildung lässt sich folglich nicht bestätigen.

Die Unterscheidung zwischen isolierten bzw. verbundenen Vereinigungen zeichnet ein ganz ähnliches Bild (Modell 3). Eine aktive Tätigkeit in verbundenen Organisationen, welche über einen hohen Anteil an überlappenden Mitgliedschaften eng mit anderen Organisationstypen verknüpft sind, scheint nicht stärker mit der Höhe des Vertrauens in die Mitmenschen in Zusammenhang zu stehen, als dies für die aktive Partizipation in eher isolierten Organisationen der Fall ist. Auch hier lassen sich keine signifikanten Unterschiede in der Effektgrösse feststellen $\left(\chi^{2}\left(1,6^{\prime} 194\right)=\right.$ $0.50, \mathrm{p}=0.48)$. Wie schon die Einteilung in brückenbildende und bindende Organisationen birgt die in Anlehnung an Paxton (2007) getroffene Unterscheidung zwischen verbundenen und isolierten Organisationen nur wenig zusätzliches Potential zur Erklärung der Vertrauensentwicklung in der Schweiz in sich.

Konzentriert man sich schliesslich auf den Aspekt der individuellen vereinstypusunabhängigen Mehrfachmitgliedschaften, offenbart sich auch hier ein positiver Effekt (siehe Tabelle 4, Modell 4). Die entscheidende Rolle multipler Mitgliedschaften wird hierbei aber noch deutlicher, wenn man zwischen der aktiven Tätigkeit in nur einer Organisation und der Partizipation in mehreren verschiedenen Tätigkeitsfeldern unterscheidet (Modell 5). Es zeigt sich, dass Personen, die lediglich in einem Verein aktiv sind, sich in ihrer Vertrauensbildung nicht systematisch von den Nicht-Engagierten unterscheiden. Mehrere Aktivitäten gleichzeitig verstärken aber den Zusammenhang zwischen Vereinsengagement und Vertrauensbildung. Diese Ergebnisse stïtzen die These, wonach eine aktive Partizipation in mehreren formellen Netzwerken die Ausbildung von Vertrauen fördert. Sie weisen darauf hin, dass entgegen weitverbreiteter Meinung die Vertrauensentwicklung in der Schweiz weniger vom Engagement in spezifischen Vereinen abhängt, sondern vielmehr dem Ausmass der persönlichen Integrationsbereitschaft geschuldet ist. Von entscheidender Bedeutung ist also nicht die Qualität der hier untersuchten einzelnen Vereinigungen, sondern die Quantität des persönlichen Engagements.

Diese Ergebnisse werden auch durch die hier nicht weiter illustrierten Berechnungen für die einzelnen Vereinstypen bestätigt. Weder bei brükkenbildenden und verbundenen, noch bei abgrenzenden und isolierten Vereinigungen führt eine Einzelaktivität zur Vertrauensbildung. Das zwischenmenschliche. Vertrauen wächst erst mit der Ausweitung des Engage- 
Tabelle 4: Regressionsmodelle für generalisiertes Vertrauen II (OLS)

\begin{tabular}{|c|c|c|c|c|}
\hline \multirow[b]{2}{*}{ Anzahl aktiver Tätigkeiten } & \multicolumn{2}{|c|}{ M4 } & \multicolumn{2}{|c|}{ M5 } \\
\hline & $0.12 * * *$ & $(0.02)$ & & \\
\hline Aktiv in einer Vereinigung & & & 0.04 & $(0.07)$ \\
\hline Aktiv in mehreren Vereinigungen & & & $0.33^{* * *}$ & $(0.06)$ \\
\hline Männlich & $-0.19 * * *$ & $(0.05)$ & $-0.185^{* * *}$ & $(0.05)$ \\
\hline Alter & $0.01^{* * *}$ & $(0.00)$ & $0.01 * * *$ & $(0,00)$ \\
\hline Ausländer & $-0.22 * *$ & $(0.08)$ & $-0.23^{* *}$ & $(0.08)$ \\
\hline Protestantisch ${ }^{b}$ & -0.00 & $(0.08)$ & -0.00 & $(0.08)$ \\
\hline Katholisch ${ }^{6}$ & -0.13 & $(0.08)$ & -0.12 & $(0.08)$ \\
\hline Andere christl. ${ }^{b}$ & $-0.44 * * *$ & $(0.13)$ & $-0.43^{* * *}$ & $(0.13)$ \\
\hline Andere nicht-christl. ${ }^{b}$ & $-0.47^{* *}$ & $(0.15)$ & $-0.45^{* *}$ & $(0.15)$ \\
\hline Bildung: Sekundärstufe $\mathrm{I}^{\mathrm{e}}$ & -0.26 & $(0.17)$ & -0.26 & $(0.17)$ \\
\hline Bildung: Sekundärstufe II ${ }^{\mathrm{c}}$ & 0.09 & $(0.16)$ & 0.09 & $(0.16)$ \\
\hline Bildung: Tertiär ${ }^{c}$ & $0.40^{*}$ & $(0.17)$ & $0.41^{*}$ & $(0.17)$ \\
\hline Mittleres Einkommen $^{\text {d }}$ & $0.17^{* *}$ & $(0.06)$ & $0.17^{* *}$ & $(0.06)$ \\
\hline Hohes Einkommen ${ }^{d}$ & 0.14 & $(0.12)$ & 0.15 & $(0.12)$ \\
\hline Einkommensangabe verweigert ${ }^{d}$ & $-0.28^{* *}$ & $(0.09)$ & $-0.28^{* *}$ & $(0.09)$ \\
\hline Lebenszufriedenheit & $0.20 * * *$ & $(0.02)$ & $0.20 * * *$ & $(0.02)$ \\
\hline Demokratiezufriedenheit & $0.17 * * *$ & $(0.01)$ & $0.17 * * *$ & $(0.01)$ \\
\hline $\begin{array}{l}\text { Treffen mit Freunden, etc.: } \\
\text { wenige Male im Monat }{ }^{\circ}\end{array}$ & $0.97 * * *$ & $(0.14)$ & $0.97 * * *$ & $(0.14)$ \\
\hline $\begin{array}{l}\text { Treffen mit Freunden: } \\
\text { mehrmals pro Woche }\end{array}$ & $1.25^{* * *}$ & $(0.14)$ & $1.26^{* * *}$ & $(0.14)$ \\
\hline Konstante & $2.35^{* * *}$ & $(0.26)$ & $2.34^{* * *}$ & $(0.26)$ \\
\hline F-Statistik & 44.6 & $* * *$ & 42.20 & $7 * * *$ \\
\hline Adj. $R^{2}$ & 0.11 & 507 & 0.11 & 9191 \\
\hline $\mathrm{N}$ & & & $6^{\prime}$ & \\
\hline
\end{tabular}

Anmerkungen: Unstandardisierte Regressionskoeffizienten, Standardfehler in Klammern; ${ }^{*} \mathrm{p}<0.05,{ }^{* *} \mathrm{p}<0.01,{ }^{* * *} \mathrm{p}<0.001$. ${ }^{a}$ Referenzkategorie: keine aktive Tätigkeit; ${ }^{b}$ Referenzkategorie: konfessionslos; ${ }^{\circ}$ Referenzkategorie: kein vollendeter Bildungsabschluss; d Referenzkategorie: niedriges Einkommen (unter 5'000 CHF); ${ }^{e}$ Referenzkategorie: höchstens wenige Male im Jahr. 
ments. Mit Blick auf die einzelnen Teststatistiken unterscheiden sich die Vereinsarten aber auch in diesen Analysen nicht systematisch voneinander (eine einzige brückenbildende Vereinigung vs, eine einzige abgrenzende Vereinigung: $\chi^{2}(1)=0.03, p=0.86$; mehrere brückenbildende Vereinigungen vs. mehrere abgrenzende Vereinigungen: $\chi^{2}(1)=1.28, p=0.26$; eine einzige isolierte Vereinigung vs. eine einzige verbundene Vereinigung: $\chi^{2}(1)=0.09, p=0.77$; mehrere isolierte Vereinigungen vs. mehrere verbundene Vereinigungen: $\chi^{2}(1)=0.64, p=0.43$ ).

Was zuletzt den Effekt der berïcksichtigten Kontrollvariablen betrifft, so zeigt sich bezüglich des Geschlechts ein schwacher Zusammenhang. Männer scheinen dabei im Vergleich zu Frauen ein etwas geringeres Vertrauen in andere Menschen zu besitzen. Für das Alter ergibt sich ein schwach positiver Einfluss. Ein hoher (tertiärer) Bildungsabschluss scheint hingegen einen deutlich grösseren Effekt auf den Grad des generalisierten Vertrauens zu haben. Bezüglich des Aspekts der persönlichen Ressourcenstärke scheinen Menschen mit einem mittleren Haushaltseinkommen, im Vergleich zu Personen mit niedrigem oder sehr hohem Einkommen, anderen im Allgemeinen mehr Vertrauen entgegenzubringen. Interessant präsentiert sich darüber hinaus der Umstand, dass Verweigerer der Einkommensangabe ein deutlich geringeres Vertrauen in andere Menschen aufweisen, als dies Personen der untersten Einkommenskategorie tun. Ebenso haben ausländische Staatsbürger ein geringeres Vertrauen in ihre Mitmenschen als Angehörige der Schweiz. Des Weiteren geht ein höherer Grad an Lebenszufriedenheit mit einem höheren generalisierten Vertrauen einher. Ein ähnlicher, wenngleich schwächerer Zusammenhang findet sich für das Vertrauen in die Herrschaftsform. Je zufriedener die Befragten mit dem Funktionieren der Demokratie sind, desto grösseres Vertrauen scheinen sie generell in andere Menschen zu besitzen. Während die Zugehörigkeit zu einer der beiden grossen Glaubensrichtungen in der Schweiz (Protestantismus, Römisch-katholisch) keinen erkennbaren Effekt hat, scheinen die kleineren Konfessionen deutlich negativ mit Vertrauen verknüpft zu sein. Betrachtet man letztlich die Teilnahme an informellen Netzwerken, so zeigt sich die Häufigkeit des Treffens mit Freunden, Verwandten oder Arbeitskollegen stark mit Vertrauen verknüpft. Personen, welche sich regelmässig informell mit Menschen treffen, die sie kennen, scheinen weitaus mehr Vertrauen in andere zu haben als Individuen, die solche direkten Kontakte eher selten oder gar nicht pflegen. 


\section{Zusammenfassung}

In der Tradition von Alexis de Tocqueville wurde in den letzten Jahren dem Engagement in zivilgesellschaftlichen Vereinigungen eine essentielle Bedeutung für die Generierung von sozialem Vertrauen zugeschrieben (vor allem Putnam 1993, 1995, 2000). Netzwerke zivilgesellschaftlichen Engagements repräsentieren hiernach horizontale Interaktionsbeziehungen, in denen der Aufbau von Normen der Gegenseitigkeit unterstützt und zwischenmenschliches Vertrauen stabilisiert wird. Darauf aufbauend stand im Fokus dieser Arbeit die empirische Analyse der Beziehung zwischen aktiver Teilnahme an solchen Interaktionsbeziehungen und dem generalisierten Vertrauen. Ziel war es, den Aspekt der Mitgliedschaft in Freiwilligenorganisationen differenzierter zu betrachten, um Unterschiede bezüglich des Organisationstyps sowie das Ausmass der Interaktionen mit unterschiedlichen Menschen innerhalb dieser Netzwerke zu berücksichtigen. Die Ergebnisse dieser Untersuchung lassen sich in drei zentralen Punkten zusammenfassen.

Erstens zeigen die Ergebnisse dieser Studie klar in Richtung einer positiven Beziehung zwischen Vereinsengagement und einem generalisierten Vertrauen in die Mitmenschen. Vereine können damit als "Schulen des Vertrauens" bezeichnet werden, denen eine wesentliche Bedeutung für den zivilgesellschaftlichen Zusammenhalt in der Schweiz zukommt.

Zweitens hält aber weder die Unterscheidung zwischen brückenbildenden und abgrenzenden Vereinigungen (Putnam 2000; Zmerli 2003, 2008) noch jene zwischen isolierten und verbundenen Organisationen (Paxton 2002, 2007) einen zusätzlichen Erkenntnisgewinn hinsichtlich der Generierung von generalisiertem Vertrauen bereit. Tatsächlich unterscheiden sich diese verschiedenen Organisationstypen nicht in ihrem Potential als Vertrauensgeneratoren. Der von Zmerli (2003) ermittelte positive Einfluss brückenbildender Freiwilligenorganisationen auf die Ausbildung von Vertrauen im Vergleich zu abgrenzenden Vereinigungen lässt sich folglich für die Schweiz nicht bestätigen. Aber auch die Befunde von Paxton (2007) zur Wirkung verbundener Organisationen werden durch unsere Analyse nicht gestützt.

Vielmehr bestätigt sich drittens der vermutete Zusammenhang zwischen persönlichen vereinstypusunabhängigen Mehrfachmitgliedschaften und einem grösseren zwischenmenschlichen Vertrauen als stärkster Effekt. Während die Mitgliedschaft in Organisationen eines einzigen Tätigkeitsfeldes nicht zwingend mit einem Mehr an Vertrauen in Verbindung ge- 
bracht werden kann, setzen Personen, die in mehr als einer Organisation partizipieren, deutlich mehr Vertrauen in ihre Mitmenschen. Vertrauen scheint somit weniger mit den speziellen Eigenschaften und Charakteristiken einer Freiwilligenorganisation, als vielmehr mit der Breite individueller Interaktionen über verschiedene Netzwerke zivilgesellschaftlichen Engagements hinweg zusammenzuhängen. Diese Ergebnisse decken sich mit den Befunden von Wollebæk/Selle (2003), wonach allein die Anzahl von Vereinsmitgliedschaften und nicht die Intensität einzelner Interaktionen in den jeweiligen Netzwerken für die Vertrauensbildung ausschlaggebend ist.

Unsere Analysen offenbaren aber auch die Notwendigkeit weiterführender Studien in diesem Bereich der Sozialkapitalforschung. Diese sollten sich bemühen, die innere Heterogenität der einzelnen Organisationen und Vereine sowie deren Verknüpfung zur Gesellschaft besser zu berücksichtigen. Da sich dies auf Grundlage repräsentativer Untersuchungen mit hohem Verallgemeinerungsgrad sehr schwierig darstellt, wären etwa Studien zur genaueren Betrachtung einzelner Freiwilligenorganisationen ein wünschenswertes Unterfangen.

\section{Literatur}

Anheier, H. und J. Kendall (2002). Interpersonal Trust and Voluntary Associations: Examining Three Approaches. British Journal of Sociology 53(3): 343-62.

Arrow, K. (1972). Gifts and exchanges. Philosophy and Public Affairs 1: 343-62.

Brehm, J. und W. Rahn (1997). Individual-Level for the Causes and Consequences of Social Capital. American Journal of Political Science 41(3): 999-1023.

Caulkins, D. (2004). Organizational Memberships and Crosscutting Ties: Bonding or Bridging Social Capital? In Prakash, S. und P. Selle (Hrsg.), Investigating Social Capital: Comparative Perspectives on Civil Society, Participation and Governance. New Delhi: Sage (162-83).

Claibourn, M. und P. Martin (2000). Trusting and Joining? An Empirical Test of the Reciprocal Nature of Social Capital. Political Behavior 22(4): 267-91. 
Coffé, H. und B. Geys (2007). Toward an Empirical Characterization of Bridging and Bonding Social Capital. Nonprofit and Voluntary Sector Quarterly 36(1): 121-39.

Coleman, J. (1990). Foundations of Social Theory. Cambridge: Harvard University Press.

Delhey, J. und K. Newton (2002). Who Trusts? The Origins of Social Trust in Seven Nations. Band 02-402, Wissenschaftszentrum Berlin für Sozialforschung.

Freitag, M. (2003). Beyond Tocqueville: The Origins of Social Capital in Switzerland. European Sociological Review 19(2): 217-32.

Freitag, M. und M. Bühlmann (2009 im Erscheinen). Crafting Trust: The Role of Political Institutions in a Comparative Perspective. Comparative Political Studies.

Freitag, M. und R. Traunmüller (2009 im Erscheinen). Spheres of Trust: An Empirical Analysis of the Foundations of Particularised and Generalised Trust. European Journal of Political Research.

Fukuyama, F. (1995). Konfuzius und Marktwirtschaft: Der Konflikt der Kulturen. München: Kindler.

Gibson, J. (2001). Social Networks, Civil Society, and the Prospects for Consolidating Russia's Democratic Transition. American Journal of Political Science 45(1): 51-68.

Hardin, R. (1996). Trustworthiness. Ethics 10(1): 26-42.

Herreros, F. (2004). The Problem of Forming Social Capital: Why Trust? New York: Palgrave Macmillan.

Hooghe, M. (2003). Voluntary Associations and Democratic Attitudes: Value Congruence as a Causal Mechanism. In Hooghe, M. und D. Stolle (Hrsg.), Generating Social Capital: Civil Society and Institutions in Comparative Perspective. New York: Palgrave Macmillan $(88-111)$.

Hooghe, M. und D. Stolle (2003). Introduction: Generating Social Capital. In Hooghe, M. und D. Stolle (Hrsg.), Generating Social Capital: Civil Society and Institutions in Comparative Perspective. New York: Palgrave Macmillan (1-18).

Howard, M. und L. Gilbert (2008). A Cross-National Comparison of the Internal Effects of Participation in Voluntary Organizations. Political Studies 56: 12-32.

Kwak, N., Shah, D. und R. Holbert (2004). Connecting, Trusting, and Participating: The Direct and Interactive Effects of Social Associations. Political Research Quarterly 57(4): 643-52. 
Letki, N. (2004). Socialization for Participation? Trust, Membership, and Democratization in East-Central Europe. Political Research Quarterly 57(4): 665-79.

Levi, M. (1998). A State of Trust. In Braithwaite, V. und M. Levi (Hrsg.), Trust and Governance. New York: Russell Sage Foundation (77-101).

Lippl, B. (2007). Soziales Engagement und politische Partizipation in Europa. In Franzen, A. und M. Freitag (Hrsg.), Sozialkapital. Grundlagen und Anwendungen. Sonderheft der Kölner Zeitschrift für Soziologie und Sozialpsychologie (420-49).

Marshall, M. und D. Stolle (2004). Race and the City. Neighborhood Context and the Development of Generalized Trust. Political Behavior 26: $125-53$.

Newton, K. (2004). Social Trust: Individual and Cross-national Approaches. Portuguese Journal of Social Science 3(1): 15-35.

Offe, C. (2001). Wie können wir unseren Mitbürgern vertrauen? In Hartmann, M. und C. Offe (Hrsg.), Vertrauen: Die Grundlage des sozialen Zusammenhalts. Frankfurt a.M. (241-94).

Paxton, P. (2002). Social Capital and Democracy: An Interdependent Relationship. American Sociological Review 67(2): 254-77.

Paxton, P. (2007). Association Memberships and Generalized Trust: A Multilevel Model Across 31 Countries. Social Forces 86(1): 47-76.

Preisendörfer, P. (1995). Vertrauen als soziologische Kategorie: Möglichkeiten und Grenzen einer entscheidungstheoretischen Fundierung des Vertrauenskonzepts. Zeitschrift für Soziologie 24: 263-72.

Putnam, R. (1993). Making Democracy Work: Civic Traditions in Modern Italy. Princeton: Princeton University Press.

- (1995). Tuning In, Tuning Out: The Strange Disappearance of Social Capital in America. Political Science and Politics 28(4): 664-83. (2000). Bowling Alone: The Collapse and Revival of American Community. New York: Simon and Schuster.

Putnam, R. und K. Goss (2001). Einleitung. In Putnam, R. (Hrsg.), Gesellschaft und Gemeinsinn: Sozialkapital im Internationalen Vergleich. Gütersloh: Bertelsmann Stiftung (15-43).

Shah, D. (1998). Civic Engagement, Interpersonal Trust, and Television Use: An Individual-Level Assessment of Social Capital. Political Psychology 19(3): 469-96.

Stadelmann-Steffen, I., Freitag, M. und M. Bühlmann (2007). Freiwilligen-Monitor Schweiz 2007. Zürich: Seismo Verlag. 
Stolle, D. (1998). Bowling Together, Bowling Alone: The Development of Generalized Trust in Voluntary Associations. Political Psychology 19(3): 497-525.

(2002). Trusting Strangers: The Concept of Generalized Trust in Perspective. Österreichische Zeitschrift für Politikwissenschaft 31(4): 397-412.

- (2003). The Sources of Social Capital. In Hooghe, M. und D. Stolle (Hrsg.), Generating Social Capital: Civil Society and Institutions in Comparative Perspective. New York: Palgrave Macmillan (19-38).

Stolle, D. und T. Rochon (1998). Are All Associations Alike? Member Diversity, Associational Type, and the Creation of Social Capital. American Behavioral Scientist 42(1): 47-65.

Stolle, D. und B. Rothstein (2007). Institutionelle Grundlagen des Sozialkapitals. In Franzen, A. und M. Freitag (Hrsg.), Sozialkapital: Grundlagen und Anwendungen. Sonderheft der Kölner Zeitschrift für Soziologie und Sozialpsychologie (113-40).

Tocqueville, A. de (1956). Democracy in America. Specially edited and abridged for the modern reader by R. Heffner. New York: New American Library of World Literature.

Traunmüller, R. (2009). Individual Religiosity, Religious Context, and the Creation of Social Trust in Germany. Schmollers Jahrbuch (Journal of Applied Social Science Studies) 129(2): 357-65.

Uslaner, E. (2002). Religion and Civic Engagement in Canada and the United States. Journal of the Scientific Study of Religion 41(2): 239-54.

Welch, M., Sikkink, D. und M. Loveland (2007). The Radius of Trust: Religion, Embeddedness and Trust in Strangers. Social Forces 86(1): $23-46$.

Whiteley, P. (2000). Economic Growth and Social Capital. Political Studies 48: 443-66.

Wollebæk, D. und P. Selle (2003). The Importance of Passive Membership for Social Capital Formation. In Hooghe, M. und D. Stolle (Hrsg.), Generating Social Capital: Civil Society and Institutions in Comparative Perspective. New York: Palgrave Macmillan (67-88).

Yamagishi, T. und M. Yamagishi (1994). Trust and Commitment in the United States and Japan. Motivation and Emotion 18(2): 129-66.

Zmerli, S. (2003). Applying the Concepts of Bonding and Bridging Social Capital to Empirical Research. European Political Science 2(3): $68-75$. 
(2008). Inklusives und Exklusives Sozialkapital in Deutschland: Grundlagen, Erscheinungsformen und Erklärungspotential eines Alternativen Theoretischen Konzepts. Baden-Baden: Nomos. 
Tabelle A1: Messung und Codierung der verwendeten Variablen

\begin{tabular}{|c|c|c|}
\hline Variable & Indikator & Codierung \\
\hline \multicolumn{3}{|l|}{ Abhängige Variable } \\
\hline $\begin{array}{l}\text { Generalisiertes } \\
\text { Vertrauen }\end{array}$ & $\begin{array}{l}\text { Frage Q02 (Schweizer Freiwilligen-Monitor 2007): "Würden Sie sa- } \\
\text { gen, dass man den meisten Menschen vertrauen kann, oder kann man } \\
\text { im Umgang mit anderen Menschen nicht vorsichtig genug sein?" }\end{array}$ & $\begin{array}{l}\text { Skala von } 0 \text { ("man kann nicht vorsichtig } \\
\text { genug sein") bis } 10 \text { ("man kann den mei- } \\
\text { sten Menschen vertrauen") }\end{array}$ \\
\hline \multicolumn{3}{|c|}{$\begin{array}{l}\text { Unabhängige Variablen } \\
\text { Aktive Tätigkeit in Freiwilligenorganisationen }\end{array}$} \\
\hline $\begin{array}{l}\text { Aktive Tätigkeit in } \\
\text { Freiwilligenorganisa- } \\
\text { tionen }\end{array}$ & \multirow{3}{*}{$\begin{array}{l}\text { Frage Q09 (Schweizer Freiwilligen-Monitor 2007): "Es gibt } \\
\text { verschiedene Arten, sich ausserhalb von Beruf und Familie irgend- } \\
\text { wo zu engagieren, beispielsweise in einem Verein oder einer Orga- } \\
\text { nisation. Ich nenne Ihnen nun verschiedene Möglichkeiten (...). } \\
\text { Uns interessiert, ob Sie in diesen Organisationen in den letzten } 12 \\
\text { Monaten Mitglied sind/waren, etwas gespendet haben oder aktiv } \\
\text { mitmachen/ mitgemacht haben oder ob davon nichts auf Sie } \\
\text { zutrifft". } \\
10 \text { vorgegebene Organisationstypen: } \\
\text { (A) Sportclubs, -vereine } \\
\text { (B) Kirche oder kirchennahe Organisationen } \\
\text { (C) Soziale/karitative/gemeinnützige Institutionen (Rotes Kreuz; } \\
\text { Glückskette) } \\
\text { (D) Kulturelle Vereine (Theatergruppen; Fastnachtsvereine) } \\
\text { (E) Interessenverband (Berufsverband, Gewerkschaft, Konsumen- } \\
\text { tenschutz, ACS, TCS, Quartiervereine usw.) } \\
\text { (F) Menschenrechte/Umweltverbände }\end{array}$} & $\begin{array}{l}\text { Dichotome Variable: Aktives Engage- } \\
\text { ment in mindestens einem der vorgege- } \\
\text { benen Organisationstypen } 1=\text { ja } 0=\text { nein }\end{array}$ \\
\hline $\begin{array}{l}\text { Brückenbildende/ } \\
\text { abgrenzende } \\
\text { Organisationen }\end{array}$ & & $\begin{array}{l}\text { Einteilung in "brückenbildende" bzw. } \\
\text { "abgrenzende Vereinigung" nach Coffé/ } \\
\text { Geys (2007); jeweils dichotom codiert: } 1 \\
=\text { aktive Tätigkeit in mind. einer Organi- } \\
\text { sation } 0=\text { keine aktive Tätigkeit }\end{array}$ \\
\hline $\begin{array}{l}\text { Isolierte/ verbundene } \\
\text { Organisationen }\end{array}$ & & $\begin{array}{l}\text { Einteilung anhand des Anteils an Mitglie- } \\
\text { dern, welche noch in anderen Organisati- } \\
\text { onstypen aktiv engagiert sind (unter } 70 \% \\
\text { Mehrfachmitglieder = isolierte Organi- } \\
\text { sation, über } 70 \% \text { Mehrfachmitglieder = } \\
\text { verbundene Organisation); jeweils } \\
\text { dichotom codiert }(1=\text { Aktive Tätigkeit in } \\
\text { mind. einer isolierten bzw. verbundenen } \\
\text { Organisation, } 0=\text { keine aktive Tätigkeit) }\end{array}$ \\
\hline
\end{tabular}


Tabelle Al (Fortsetzung): Messung und Codierung der verwendeten Variablen

\begin{tabular}{|c|c|c|}
\hline Variable & Indikator & Codierung \\
\hline $\begin{array}{l}\text { Ausmass der Tätigkeit } \\
\text { in Freiwilligen- } \\
\text { organisationen }\end{array}$ & $\begin{array}{l}\text { (G) Politische Parteien } \\
\text { (H) Öffentliche Dienste (Feuerwehr, Samariter, usw.) } \\
\text { (I) Spiel-/Hobby-/Freizeitvereine } \\
\text { (J) Politisches oder öffentliches Amt (Parlament, gewähltes Mitglied } \\
\text { einer Kommission, eines Gerichts, Vormund, etc.) } \\
\text { (nur aktive Teilnahme berücksichtigt) }\end{array}$ & $\begin{array}{l}\text { Metrische Variable für die Anzahl der } \\
\text { aktiven Mitgliedschaften in verschie- } \\
\text { denen Organisationstypen; Kategoriale } \\
\text { Variable für die Mehrfachmitgliedschaft: } \\
0=\text { keine aktive Tätigkeit } \\
1 \text { = aktive Tätigkeit in einem Organisa- } \\
\text { tionstyp } \\
2=\text { aktive Tätigkeit in mehr als einem } \\
\text { Organisationstyp }\end{array}$ \\
\hline \multicolumn{3}{|l|}{ Kontrollvariablen } \\
\hline Geschlecht & $\begin{array}{l}\text { Frage Sd03 (Schweizer Freiwilligen-Monitor 2007) nach dem Ge- } \\
\text { schlecht der befragten Person }\end{array}$ & $\begin{array}{l}\text { Dichotome Variable: } \\
1=\text { männlich } 0=\text { weiblich }\end{array}$ \\
\hline Alter & $\begin{array}{l}\text { Frage Sd01 (Schweizer Freiwilligen-Monitor 2007): "Was haben Sie } \\
\text { für einen Jahrgang" }\end{array}$ & $\begin{array}{l}\text { Metrische Variable für das Alter: } \\
\text { Jahr des Interviews - Geburtsjahr }\end{array}$ \\
\hline Religiosität & $\begin{array}{l}\text { Frage Sd22 (Schweizer Freiwilligen-Monitor 2007): "Welches ist } \\
\text { Ihre Konfession oder Religion?" }\end{array}$ & $\begin{array}{l}\text { Kategoriale Einteilung: } \\
0=\text { konfessionslos } \\
1=\text { Protestantisch oder reformiert } \\
2=\text { Römisch-katholisch } \\
3=\text { christkatholisch und andere } \\
\text { christliche Konfessionen } \\
4=\text { andere Religionen (Jüdisch, } \\
\text { Muslimisch, andere Religion) }\end{array}$ \\
\hline Lebenszufriedenheit & $\begin{array}{l}\text { Frage Q04 (Schweizer Freiwilligen-Monitor 2007): "Wie zufrieden } \\
\text { sind Sie alles in allem mit Threm gegenwärtigen Leben?" }\end{array}$ & $\begin{array}{l}\text { Skala von } 0 \text { ("sehr unzufrieden") bis } \\
10 \text { ("sehr zufrieden") }\end{array}$ \\
\hline
\end{tabular}


Tabelle Al (Fortsetzung): Messung und Codierung der verwendeten Variablen

\begin{tabular}{|c|c|c|}
\hline Variable & Indikator & Codierung \\
\hline $\begin{array}{l}\text { Zufriedenheit mit } \\
\text { Demokratie }\end{array}$ & $\begin{array}{l}\text { Frage Q08 (Schweizer Freiwilligen-Monitor 2007): "Wie sind Sie } \\
\text { alles in allem mit der Art und Weise, wie die Demokratie in der } \\
\text { Schweiz funktioniert, zufrieden?" }\end{array}$ & $\begin{array}{l}\text { Skala von } 0 \text { ("uberhaupt nicht zufrieden") } \\
\text { bis } 10 \text { ("sehr zufrieden") }\end{array}$ \\
\hline Bildung & $\begin{array}{l}\text { Frage Sd21 (Schweizer Freiwilligen-Monitor 2007): "Welches ist } \\
\text { die höchste Ausbildung, wo Sie mit einem Zeugnis oder einem } \\
\text { Diplom abgeschlossen haben?" }\end{array}$ & $\begin{array}{l}\text { Kategoriale Einteilung (analog zu Stadel- } \\
\text { mann-Steffen et al. 2007): } \\
0=\text { keine abgeschlossene Ausbildung } \\
\text { (keine Ausbildung abgeschlossen, ist } \\
\text { noch in obligatorischer Schule) } \\
1=\text { Sekundärstufe I (Obligatorische } \\
\text { Schule, Anlehre, Diplommittelschule, } \\
\text { allgemein bild. Schule) } \\
2=\text { Sekundärstufe II.(Berufslehre, Voll- } \\
\text { zeitberufsschule, Berufsmaturität, Matu- } \\
\text { ritätsschule, Lehrseminar (alt), 1-2 jäh- } \\
\text { rige Handelsschule/Haushaltslehrjahr) } \\
3=\text { Tertiär (Höhere Berufsausbildung mit } \\
\text { Meisterdiplom, Eidg. Fachausweis, Tech- } \\
\text { niker oder Fachschule, höhere Fach- } \\
\text { schule, HTL, HMV, Universität) }\end{array}$ \\
\hline
\end{tabular}


Tabelle A1 (Fortsetzung): Messung und Codierung der verwendeten Variablen

\begin{tabular}{|c|c|c|}
\hline Variable & Indikator & Codierung \\
\hline $\begin{array}{l}\text { Verfügbares Haushalts- } \\
\text { einkommen }\end{array}$ & $\begin{array}{l}\text { Frage Sd23 (Schweizer Freiwilligen-Monitor 2007): "Nun sollte } \\
\text { ich noch das ungefähre verfügbare Monats-Einkommen von Ihrem } \\
\text { Haushalt notieren. Könnten Sie bitte die Einkommen von allen } \\
\text { Personen in Ihrem Haushalt zusammenzählen, dass Ihnen nach } \\
\text { Abzug der Sozialversicherungsbeiträge zu Verfügung steht?" }\end{array}$ & $\begin{array}{l}\text { Einteilung in Einkommenskategorien } \\
\text { (vgl. Stadelmann-Steffen et al. 2007): } \\
1=\text { niedrig (unter 5'000 CHF), } \\
2=\text { mittel (5'000-11'000 CHF), } \\
3=\text { hoch (über } 11^{\prime} 000 \mathrm{CHF} \text { ) } \\
4=\text { keine Angabe/ verweigert }\end{array}$ \\
\hline $\begin{array}{l}\text { Einbindung in } \\
\text { informelle Netzwerke: } \\
\text { Häufigkeit des } \\
\text { Treffens mit Freunden, } \\
\text { Verwandten und } \\
\text { Arbeitskollegen }\end{array}$ & $\begin{array}{l}\text { Frage Q01 (Schweizer Freiwilligen-Monitor 2007): "Wie oft } \\
\text { treffen Sie sich mit Freunden, Verwandten oder Arbeitskollegen } \\
\text { ausserhalb der Arbeit?" }\end{array}$ & $\begin{array}{l}\text { Kategoriale Einteilung: } \\
1=\text { höchstens wenige Male im Jahr } \\
\text { (Nie oder weniger als einmal im Monat) } \\
2=\text { mindestens monatlich (Einmal pro } \\
\text { Monat/Mehrmals pro Monat/Einmal die } \\
\text { Woche) } \\
3=\text { mehrmals die Woche (Mehrmals pro } \\
\text { Woche/Jeden Tag) }\end{array}$ \\
\hline
\end{tabular}




\section{Associations as Schools of Trust? An Empirical Analysis of the Swiss Civil Society}

According to the theory of Alexis de Tocqueville, associational life within civil societies is considered to be of essential importance for the generation of interpersonal trust. In this article, we take a closer look at the relationship between associational membership and generalized trust. We present an empirical analysis focusing on Switzerland and encompassing participation in different types of associations (bridging vs. bonding and isolated vs. connected associations) as well as multiple participation irrespective of the type of association. Empirical analyses on the basis of data obtained by means of the Schweizer Freiwilligen-Monitor of 2007 lead us to the conclusion that in contrast to widely-held beliefs the generation of trust in Switzerland does not so much depend on membership in specific associations but rather on the personal willingness to integrate. This means that social trust is built for the most part depending on the quantity of personal voluntary engagement, and scarcely depends on the quality of the associations joined.

\section{Les associations comme écoles de confiance? Une analyse empirique de la société civile en Suisse}

Dans la tradition d'Alexis de Tocqueville les associations bénévoles sont d'une importance essentielle pour le développement de la confiance interpersonnelle. Le but de la présente contribution est d'examiner d'une manière plus nuancée la relation entre engagement bénévole au sein des associations et confiance généralisée. Il s'agira ensuite de présenter une analyse empirique pour la Suisse en comparant non seulement l'engagement dans différents types d'associations (des associations de « cohésion » ou de « liaison » ainsi que « isolées » et « reliées ») mais aussi l'intégration associative qui est indépendante du type d'association. L'exploitation des données de l'Observatoire suisse du bénévolat 2007 montre à l'évidence que - contrairement aux expectations - le développement de la confiance en Suisse est moins le résultat d'un engagement dans des associations spécifiques que de la propension individuelle à l'intégration. Ce n'est donc pas la qualité de certains types d'associations examinées dans les présentes analyses, mais la quantité de l'engagement individuel qui est d'une importance cruciale pour le développement de la confiance. 
Markus Freitag ist Professor und Inhaber des Lehrstuhls für Vergleichende Politik am Fachbereich Politik- und Verwaltungswissenschaft an der Universität Konstanz. Seine Schwerpunkte liegen in der Analyse des Sozialkapitals und der Zivilgesellschaft, in der Erforschung politischer Institutionen, in der vergleichenden Policy-Forschung und in der subnationalen Demokratieanalyse. Neuere Forschungsarbeiten sind vor kurzem erschienen in British Journal of Political Science, Comparative Political Studies, European Journal of Political Research, European Union Politics, Governance, Journal of European Public Policy, Publius oder West European Politics. Seine jüngste Buchpublikation trägt den Titel: Die Demokratien der deutschen Bundesländer: Politische Institutionen im Vergleich (herausgegeben mit Adrian Vatter, Verlag UTB).

Korrespondenzadresse: Department of Politics and Management, University of Konstanz, PO Box 84, D-78457 Konstanz, Deutschland. E-Mail: markus.freitag@,uni-konstanz.de.

Nicolas Grießhaber besitzt einen BA-Abschluss in Politik- und Verwaltungswissenschaften der Universität Konstanz. Im Herbst 2009 absolvierte er seinen Master of Science in International Strategy and Economics an der University of St Andrews. Seine Abschlussarbeit beschäftigte sich dabei mit dem Einfluss brückenbildender und bindender Netzwerke auf Korruption.

Korrespondenzadresse: Department of Politics and Management, University of Konstanz, PO Box 84, D-78457 Konstanz, Deutschland. E-Mail: nicolas,griesshaber@uni-konstanz. de.

Richard Traunmüller ist wissenschaftlicher Mitarbeiter des Lehrstuhls für Vergleichende Politik am Fachbereich Politik- und Verwaltungswissenschaft an der Universität Konstanz. Seine Forschungsschwerpunkte liegen in der Sozialkapitalforschung, der Politischen Soziologie und Kulturforschung, sowie den quantitativen Methoden. Bisherige Veröffentlichungen sind erschienen in Berliner Journal für Soziologie, European Journal of Political Research, Schmollers Jahrbuch und Zeitschrift für Vergleichende Politikwissenschaft.

Korrespondenzadresse: Department of Politics and Management, University of Konstanz, PO Box 84, D-78457 Konstanz, Deutschland. E-Mail: richard.traunmueller@uni-konstanz. de. 
